\title{
Validation of micro-meso electrical relations for laminates with varying anisotropy
}

\author{
Lakshmi Selvakumaran $^{\mathrm{a}}$ and Gilles Lubineau ${ }^{\mathrm{b}}$ \\ King Abdullah University of Science and Technology (KAUST) \\ Physical Science and Engineering Division, COHMAS Laboratory Thuwal 23955-6900. Saudi Arabia \\ alakshmi.selvakumaran@kaust.edu.sa, bgilles.lubineau@kaust.edu.sa
}

Keywords: Transverse cracking, Mesoscale homogenization, Electrical tomography, Sensitivity

\begin{abstract}
For electrical impedance tomography (EIT) to be useful in monitoring transverse cracks in composites, it is imperative to establish the relation between conductivity and cracking density. Micro to meso scale homogenization has been developed for classical carbon fiber reinforced polymer (CFRP) laminate which provides such a relationship. However, we have shown in previous studies that the detectability of transverse cracks in such CFRP, which are characterized by very anisotropic electrical properties, is poor. Then, it is better to lower the electrical anisotropy, which can be achieved by various technologies including doping the polymeric resin by conductive nanoparticles. However, the validity of mesoscale homogenization for laminates with such low anisotropy has not been tested before. Here, we show that the mesoscale damage indicator is intrinsic for composites with varying anisotropy.
\end{abstract}

\section{Introduction}

Ability to track damage in composites while in operation is crucial to successful application of such materials in critical structures. This is because composites can degrade without any visible signs on the surface, thereby compromising the integrity and safety of the structure. This requires structural health monitoring techniques that can be used in-situ and in real time. EIT is one such technique that uses potential measurements obtained from the surface of the laminate through electrodes to construct the internal conductivity of the material. Provided that the damage causes the conductivity of the material to change significantly, this can be used to correlate the level and type of damage within the material.

Laminated composites exhibit mechanisms of degradation at two different levels as shown in Fig. 1 [1]: i) intralaminar, where the degradation mechanisms occur within the ply and includes (a) diffuse ply damage [2,3], (b) transverse cracking, (c) fiber breaking [4, 5] and (d) local delamination [6, 7, 8] (ii) interlaminar, where the mechanisms are focussed at the interface between the two plies and it includes (a) diffuse interface damage and (b) macroscopic delamination. To detect these different forms of damage, we need to solve two inverse problems associated with EIT. The first one involves the reconstruction of the conductivity map from the surface potential measurements and the second one involves interpreting the obtained conductivity map in terms of the different damage mechanisms. Both of these problems are highly ill-posed and as such requires specific regularization techniques to obtain a unique solution. The mesoscale homogenization of transverse cracks can regularize the first inverse problem and can be used to interpret the level of transverse cracks in the second problem as well. Through mesoscale homogenization the cracked ply can be equivalently represented by a homogeneous ply whose conductivity is modified by the damage indicator. The damage indicator is shown to be a function of the cracking density and is intrinsic to the damaged ply (independent of the geometric parameters of the surrounding plies) $[9,10]$. This is the major point that, by itself, justifies using mesoscale approaches as a reduced model for the description of transverse cracking: it is indeed valid to replace a cracked ply by an equivalent damaged ply, with reduced electrical conductivity at the mesoscale. However, the application of EIT to detect transverse cracks also requires that the voltage measurements are sensitive to the presence of such cracks. Then comes the challenge of detectability: 
how can we ensure that voltage measurements from the outer skins will be sensitive enough to probe the transverse cracks in the inner plies?

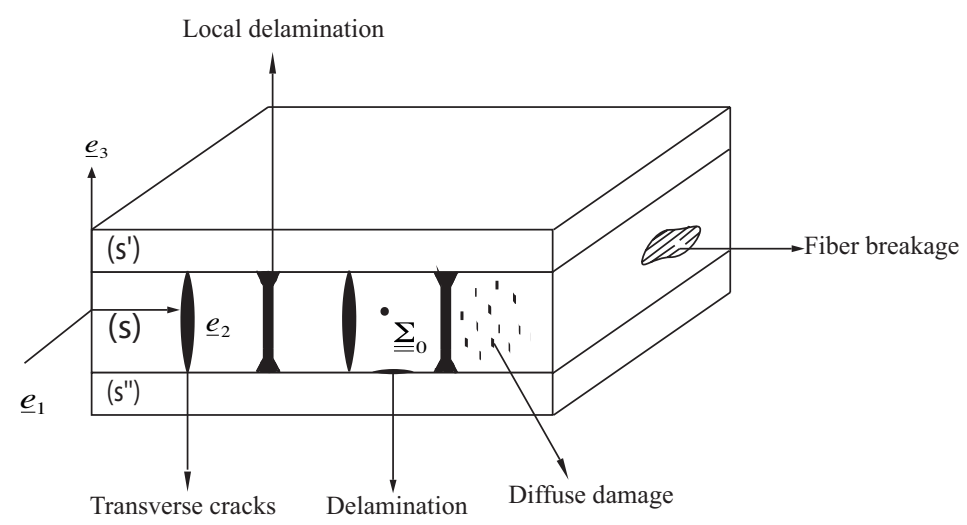

Fig. 1: Different types of damage in CFRP laminate

Classical CFRP laminates display very high level of anisotropy and are also characterized by poor detectability [11]. That means transverse cracks in such laminates are hard to detect. Reducing the anisotropy ratio has been shown to improve the detectability. Anisotropy ratio can be reduced by improving the conductivity of the matrix by doping them with conductive nanoparticles. However, at such low anisotropy ratios, we are yet to investigate whether the intrinsic feature of the relationship between equivalent damage and the cracking rate still holds. This is important as the mesoscale homogenization is valid only if the obtained description is intrinsic. By intrinsic, we mean that the developed damage indicator is independent on the material and geometric properties of the surrounding plies. The current paper focusses on validating the intrinsic property for laminates with varying anisotropy ratio.

We begin with the description of the electrical behavior of a composite ply and the classification of the different families of composite laminates in the first section. Also, we describe the mesoscale homogenization of transverse cracks followed by the definition of detectability of transverse cracks. In the second section we discuss the study of the intrinsic feature of the mesoscale homogenization for different anisotropic ratios.

\section{Mesoscale homogenization and detectability of transverse cracks}

Electrical behavior of a single composite ply: The electrical behavior of composite plies is usually highly anisotropic $[12,13,14]$. the conductivity tensor $\underline{\Sigma}^{\circ}$ of a composite ply, before the development of any damage, is assumed to be a diagonal operator [15]:

$$
\underline{\underline{\Sigma}}^{\circ}=\left[\begin{array}{ccc}
\Sigma_{1}^{\circ} & 0 & 0 \\
0 & \Sigma_{2}^{\circ} & 0 \\
0 & 0 & \Sigma_{3}^{\circ}
\end{array}\right]_{\left(e_{1}, e_{2}, e_{3}\right)},
$$

where $\left(e_{1}, e_{2}, e_{3}\right)$ denotes the local coordinate system with respect to the ply, $e_{1}$ denotes the fiber direction, $e_{2}$ and $e_{3}$ represent the transverse and through-thickness directions, respectively.

In CFRPs, carbon fibers are electrically conductive in nature with conductivity in the order of $10^{5} \mathrm{~S} / \mathrm{m}[16,17]$ while the polymer matrix is a very good insulator with conductivity in the range of $10^{-13}-10^{-10} \mathrm{~S} / \mathrm{m}[18,19]$. The conductivity in the longitudinal direction is mainly due to the conductivity along the fibers. We observe conductivity along the transverse and through-thickness directions from contacts between fibers. However, the presence of matrix-rich interlamina makes the ply more conductive along the in-plane transverse direction than in the thickness direction. Thus, conductivity 
Table 1: Typical electrical conductivity values for neat and doped laminates

\begin{tabular}{|c|c|c|c|c|c|c|c|c|}
\hline Material & Nanomat. & Vol. fr. & $\Sigma_{1}^{\circ}(\mathrm{S} / \mathrm{m})$ & $\Sigma_{2}^{\circ}(\mathrm{S} / \mathrm{m})$ & $\Sigma_{3}^{\circ}(\mathrm{S} / \mathrm{m})$ & $\lambda$ & $\lambda_{t}$ & Ref. \\
\hline \hline CFRP & - & 0.4 & 3700 & 0.666 & 0.0592 & 5555.56 & 11.25 & {$[14]$} \\
\hline & - & 0.47 & 4600 & 5.06 & 1.012 & 909.09 & 5 & {$[14]$} \\
\hline & - & 0.63 & 2439 & 58.8 & 1.02 & 41.48 & 57.65 & {$[21]$} \\
\hline & - & 0.66 & 1639.3 & 41.67 & 30.3 & 39.3 & 1.38 & {$[21]$} \\
\hline & - & 0.62 & 5500 & 203.5 & 20.9 & 27.02 & 9.74 & {$[14]$} \\
\hline Doped CFRP & $3 \%$ CB-CC & 0.654 & 1961 & 90.9 & 55.56 & 21.6 & 2.6 & {$[21]$} \\
\hline & $1 \%$ CNT & - & 158.73 & 17.47 & 2.11 & 9.1 & 8.28 & {$[22]$} \\
\hline Doped GFRP & $2 \%$ CB & 0.62 & $7.69 \times 10^{-4}$ & $1.68 \times 10^{-4}$ & $7.9 \times 10^{-6}$ & 4.6 & 21.27 & {$[23]$} \\
\hline & 2\% CB-CC & 0.6 & $3.89 \times 10^{-3}$ & $1.2 \times 10^{-3}$ & $3 \times 10^{-5}$ & 3.24 & 40 & {$[23]$} \\
\hline & $7 \%$ CB & 0.3 & 1.25 & 0.4 & 1.11 & 3.125 & 0.36 & {$[24]$} \\
\hline
\end{tabular}

ratio, $\lambda$, which we define as the ratio of the longitudinal to the in-plane transverse conductivity, is large for a CFRP. Doping the epoxy with conductive nanoparticles such as carbon nanotubes (CNT) or carbon black particles improves the conductivity of the epoxy, thereby improving the conductivity in the transverse direction. This decreases $\lambda$. In the case of Glass-Fiber Reinforced Plastic (GFRP), both the matrix and the glass fibers [20] are insulators. Using nano-modified epoxy improves the conductivity of the matrix, thus resulting in more conductivity in the transverse direction. This decreases $\lambda$ as well.

We also define $\lambda_{t}$ which is the ratio of the transverse and through-thickness conductivity. Typical values of $\lambda$ and $\lambda_{t}$ for neat and nano-doped laminates are shown in Table 1, respectively. From Table 1 , one observes that $\lambda$, varies from 3 to 6000 and $\lambda_{t}$ varies between $0.3-60$. In terms of the different families of composites, Fig. 2 shows that neat CFRPs exhibit high anisotropy whereas nano-doped GFRPs exhibit the least.

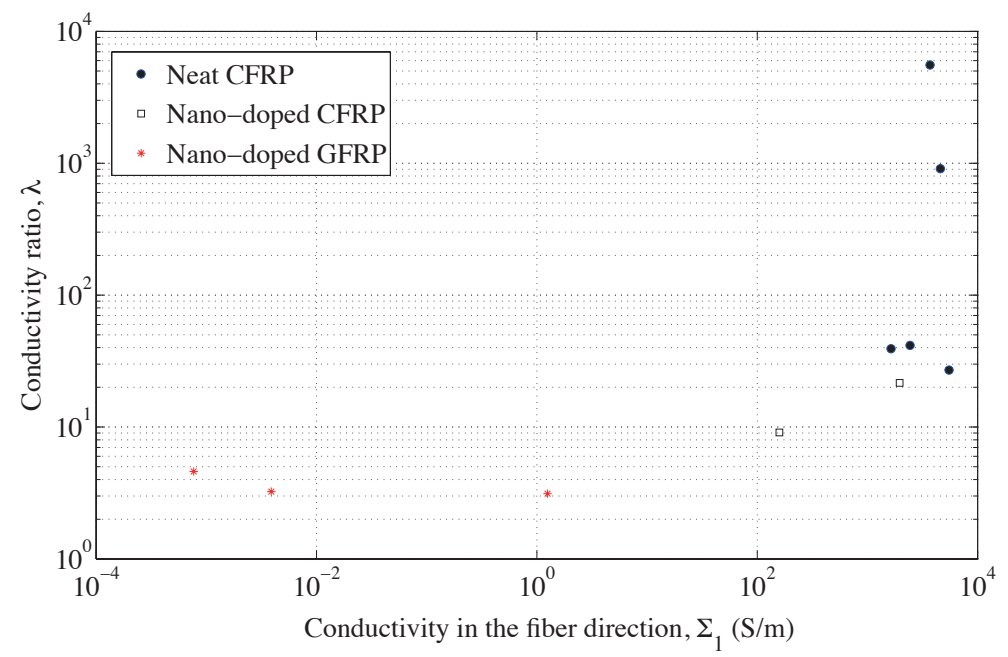

Fig. 2: Variation of $\lambda$ in typical unidirectional plies (with references)

Mesoscale modeling of transverse cracks: Transverse cracks are cracks that are parallel to the fibers and run through the thickness of the ply. They represent the primary source of degradation in the transverse direction. These cracks are usually periodically distributed. They are thus monitored by counting the number of cracks over a gage length and can be represented by a dimensionless cracking 
density:

$$
\rho=\frac{H}{L},
$$

where $H$ is the thickness of the cracked ply and $L$ is the average spacing between the cracks. Experiments have shown that the level of transverse cracking saturates at $\rho=0.8-1.2$ beyond which local delamination initiates at the tips of these cracks $[6,7,8]$.

These microcracks are discrete discontinuities and mesoscale homogenization is a viable approach to represent the effect of these defects globally at the ply scale. This is done through the introduction of global variables that relate $\rho$ to properties at the ply level such as conductivity/stiffness under the condition that the energy is equivalent between the two scales. In $[9,10]$, an electrical mesomodel was developed based on homogenization to describe the effect of $\rho$ on the electrical conductivity of the laminate. The cracked ply is equivalently represented by a homogeneous ply with electrical properties modified by the damage indicator, $d_{e}$ (Fig. 3 (a)).

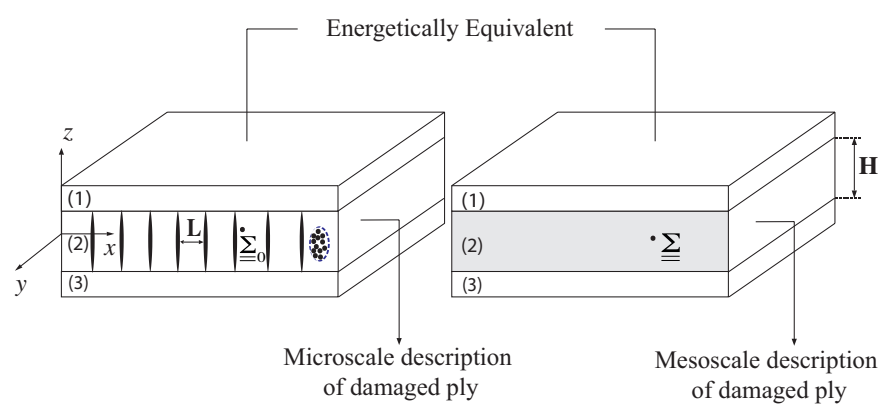

(a)

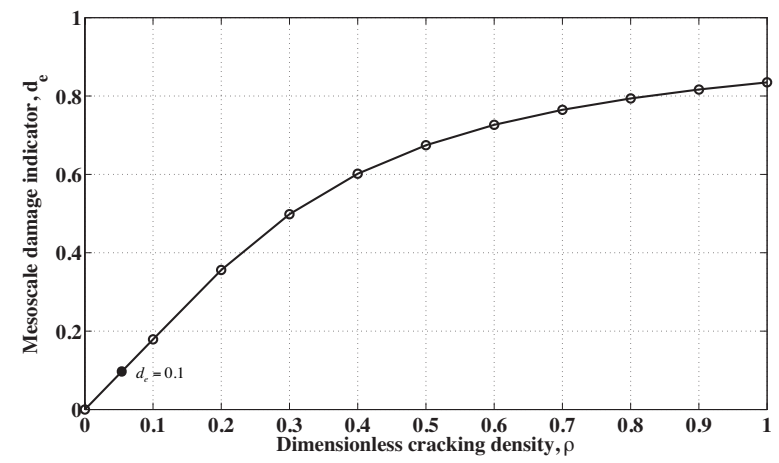

(b)

Fig. 3: (a) Mesoscale Homogenization, (b) Relationship between $d_{e}$ and $\rho$

The transverse cracks modify the transverse electrical conductivity $\Sigma_{2}=\Sigma_{2}^{\circ}\left(1-d_{e}\right)$ of the ply with a level of change directly related to the level of cracking, $\rho$. Fig. 3 (b) shows $d_{e}$ as a function of the dimensionless cracking density $\rho$ for a standard cross-ply CFRP laminate with $\Sigma_{1}=5500 \mathrm{~S} / \mathrm{m}$, $\Sigma_{2}=203.5 \mathrm{~S} / \mathrm{m}, \Sigma_{3}=20.9 \mathrm{~S} / \mathrm{m}$. This damage indicator was shown to be intrinsic, meaning that the evolution of $d_{e}$ depends only on the cracked state of the ply and not on the geometric and material parameters of the surrounding plies. This makes the model generic, in that it can be applied to any laminate structure.

Detectability of transverse cracks through electrical measurements: Successful application of EIT to identify damage in composites is only possible if the damage is detectable through the surface voltage measurements, i.e. the measurements are sensitive to the different damage mechanisms. Since conductivity of composites is anisotropic, the sensitivity of the measurements with respect to the various damage mechanisms are different and direction-dependent. Transverse cracks are said to be detectable if the change in the electrical energy due to the introduction of these cracks exceeds (i) the resolution of the measuring instruments and (ii) the level of noise in the measurements. In [11] detectability of transverse cracks in laminates with different anisotropy ratio was quantified by calculating sensitivity as the relative change in voltage produced for $10 \%$ change in transverse conductivity. We refer the reader to [11] for a complete description of sensitivity and experimental set-up.

For the cross-ply and quasi-isotropic laminates, it was shown that sensitivity reduces drastically with increasing conductivity ratio (Fig. 4). If we consider a conservative minimum threshold of $10 \%$ change in the voltage measurements for accurate/reliable detection, then the maximum feasible $\lambda$ reduces to 3. This means that, when going to applications, it is very likely that even CFRPs will have 
to be doped with nanoparticles to make the electrical anisotropy much less than usual. However, the intrinsic nature of the damage indicator obtained through the mesoscale homogenization has not been validated for composites with varying anisotropic ratio. This is important as the mesoscale analysis is valid only if the damage variable is intrinsic. In the next section, we try to study the intrinsic nature of the mesoscale damage indicator for samples with conductivities as described in Table 1.

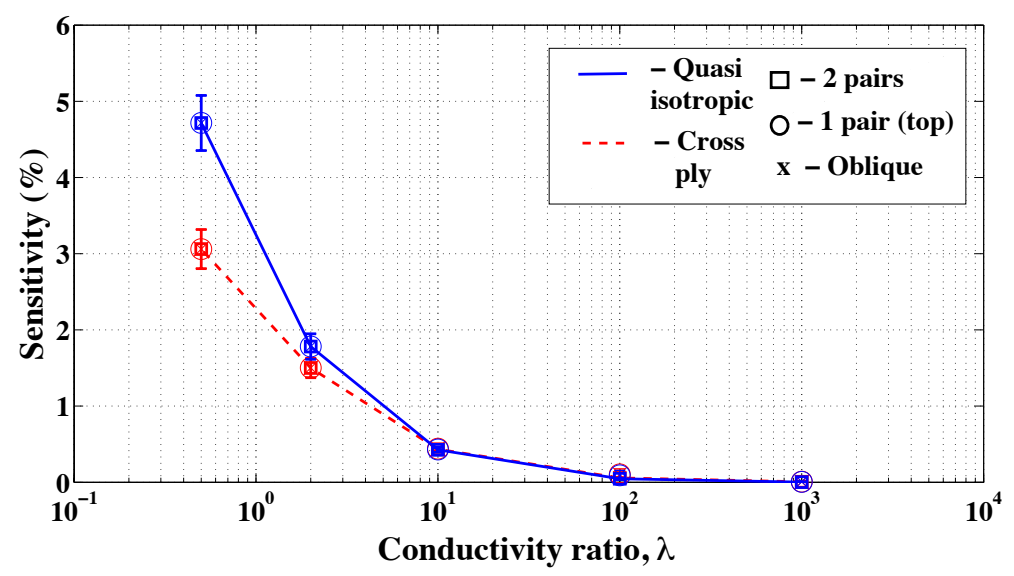

Fig. 4: Relationship between sensitivity and $\lambda$ for $10 \%$ change in transverse conductivity of the $90^{\circ}$ layer in various types of laminates (cross-ply and quasi-isotropic) and various electrical current configurations (2 pair, 1 pair and oblique) (see [11] for details)

\section{Study: Validation of intrinsic feature for different composites}

The damage parameter, $d_{e}$ by definition is dependent on the material parameters of the ply $P$, the dimensionless cracking density $\rho$ and all the geometrical parameters of the surrounding plies $\left\{\Omega^{\prime}, \Omega^{\prime \prime}\right\}_{\text {geom }}$ (stacking sequence, orientation and thickness). Thus, a general expression for $d_{e}$ would be

$$
d_{e}\left(\rho ; P ;\left\{\Omega^{\prime}, \Omega^{\prime \prime}\right\}_{\text {geom }}\right)
$$

However for the mesoscale description to be valid, $d_{e}$ must be independent of $\left\{\Omega^{\prime}, \Omega^{\prime \prime}\right\}_{\text {geom }}$ and this has been validated for a classical CFRP laminate with an anisotropic ratio of 27 in $[9,10]$. Now we check the intrinsic feature for the other families of laminates described in section marked with varying anisotropic ratios. The problem description and solution method can be found in $[9,10]$ in detail.

Influence of ply thickness: For inner plies, the thickness of the bottom $\left(H^{\prime}\right)$ and the top plies $\left(H^{\prime \prime}\right)$ were varied as $H^{\prime}=H^{\prime \prime}=[0.5,1,2,4,8] . H$ and the behavior of the damage variable with conductivities described in Table 1 is determined. Fig. 5 shows that the damage indicator is independent of the thickness of the surrounding plies.

Also, one can see that the damage curves are different for different materials. With careful observation one can see that this difference is due to the value of $\lambda_{t}$. The higher $\lambda_{t}$, higher the damage at a given cracking density. This is because when $\lambda_{t}$ is smaller, the applied energy diffuses more along the through-thickness direction and hence the damage gets smaller.

Influence of orientation: We study the influence of the orientation of the surrounding plies by varying the orientation of the two outer plies $\left(\theta^{\prime}, \theta^{\prime \prime}\right)$ as $\theta^{\prime} \in\left[0^{0}, 30^{\circ}, 45^{\circ}, 60^{\circ}, 90^{\circ}\right]$ and $\theta^{\prime \prime} \in\left[0^{0}, 30^{\circ}\right.$, $\left.45^{0}, 60^{\circ}, 90^{\circ}\right]$. From Fig. 6 , we can observe that the damage indicator is independent of the orientation 


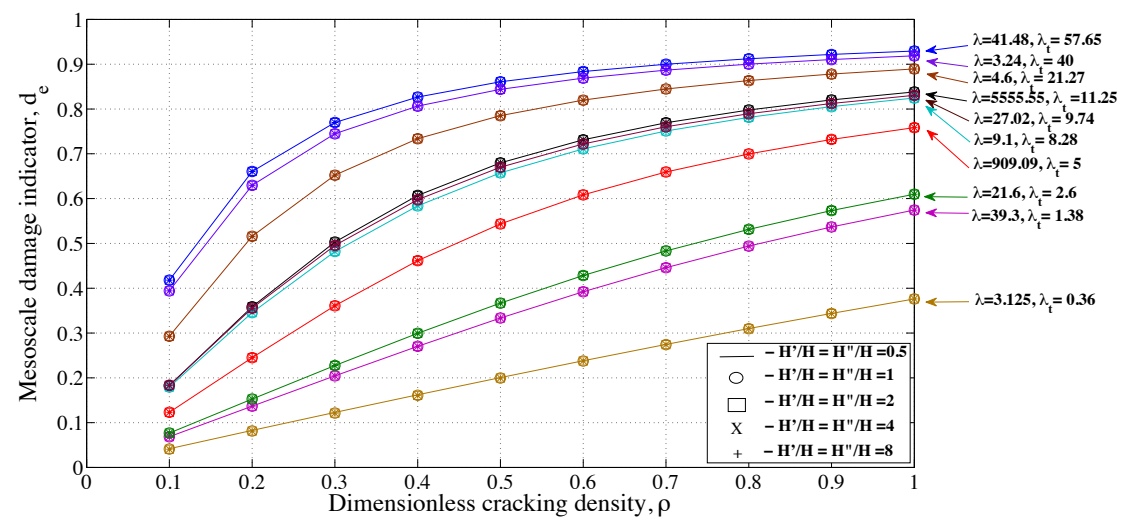

Fig. 5: Influence of thickness of the outer plies on the damage indicator of degraded inner ply

of surrounding plies as well. Table 2 provides a quantitative measure of the variations in the damage indicator for $\rho=0.2$. It can be seen that the maximum variation is $27 \%$. Although not shown in the paper, the relative variation decreases for increasing $\rho$.

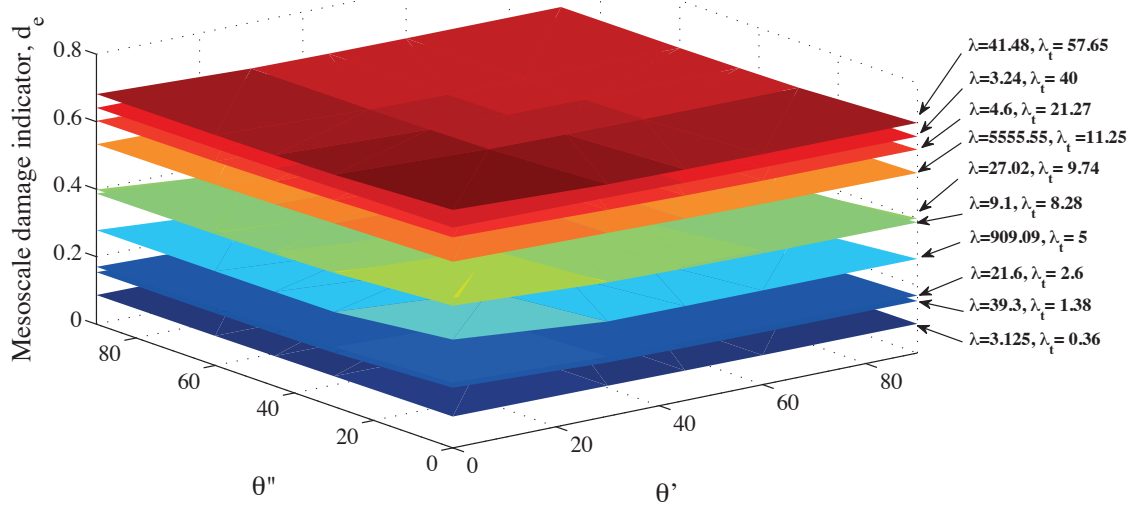

(a) $\rho=0.2$

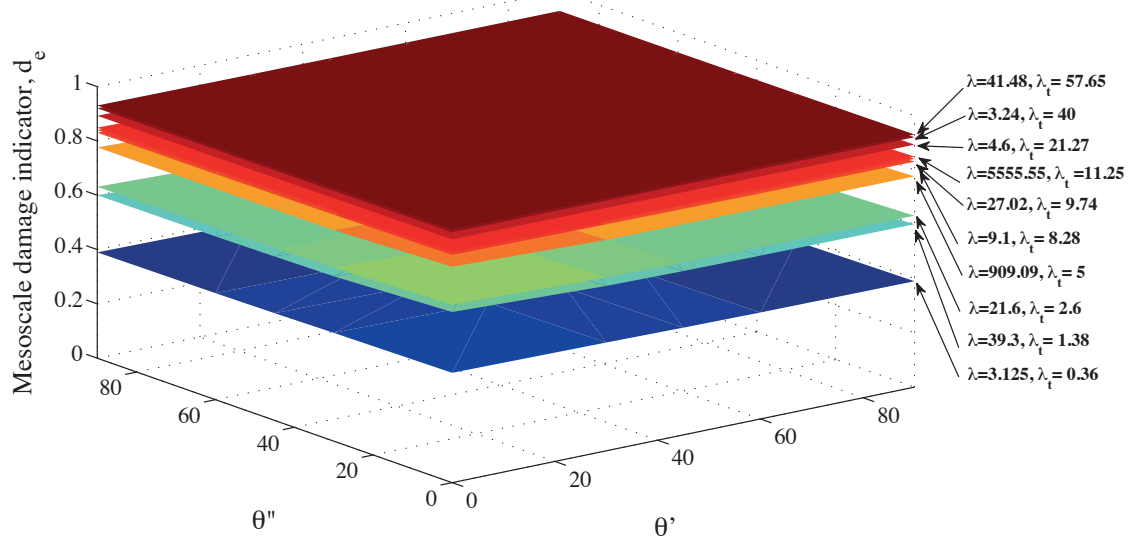

(b) $\rho=1$

Fig. 6: Influence of orientation of the outer plies on the damage indicator of degraded inner ply 
Table 2: Relative variation in the damage indicator with varying orientations of the surrounding plies for $\rho=0.2$

\begin{tabular}{|c|c|c|c|c|}
\hline$\lambda$ & $\lambda_{t}$ & $d_{\max }$ & $d_{\min }$ & Rel.variation (\%) \\
\hline \hline 5555.55 & 11.25 & 0.445 & 0.358 & 21.62 \\
\hline 909.09 & 5 & 0.321 & 0.244 & 27.1 \\
\hline 41.48 & 57.65 & 0.705 & 0.659 & 6.8 \\
\hline 39.3 & 1.38 & 0.178 & 0.136 & 26.6 \\
\hline 27.02 & 9.74 & 0.421 & 0.355 & 17 \\
\hline 21.6 & 2.6 & 0.193 & 0.152 & 23.9 \\
\hline 9.1 & 8.28 & 0.395 & 0.344 & 13.8 \\
\hline 4.6 & 21.27 & 0.552 & 0.516 & 6.7 \\
\hline 3.24 & 40 & 0.653 & 0.630 & 3.6 \\
\hline 3.125 & 0.36 & 0.093 & 0.081 & 13.3 \\
\hline
\end{tabular}

\section{Conclusion}

We have shown that the mesoscale damage indicator derived for composite laminates with different anisotropic ratios is intrinsic with respect to the geometrical properties of the surrounding plies. We have also shown that the damage curves are dependent on not only $\lambda$ but also on $\lambda_{t}$. The highlight of the current work is that it is shown that all composite laminates can be homogenized under the mesoscale assumption. This is an important result as the mesoscale homogenization helps in regularizing the inverse problem associated with EIT.

\section{References}

[1] G. Lubineau, A pyramidal modeling scheme for laminates-identification of transverse cracking, International Journal of Damage Mechanics 19 (4) (2010) 499--518.

[2] H. Zhang, M. L. Ericson, J. Varna, L. A. Berglund, Transverse single-fibre test for interfacial debonding in composites: 1. Experimental observations, Composites Part A 28 (1997) 309--315.

[3] F. Lagattu, M. C. Lafarie-Frenot, Variation of PEEK matrix crystallinity in APC-2 composite subjected to large shearing deformations, Composites Science and Technology 60 (2000) 605-612.

[4] C. Beetz, The analysis of carbon fibre strength distributions exhibiting multiple modes of failure, Fibre Science and Technology 16 (1982) 45--59.

[5] Z. Chi, T. W. Chou, G. Shen, Determination of single fibre strength distribution from fibre bundle testings, Journal of Materials Science 19 (10) (1984) 3319--3324.

[6] J. Nairn, S. Hu, The initiation and growth of delaminations induced by matrix microcracks in laminated composites, International journal of fracture 57 (1992) 1--24.

[7] S. Ogihara, N. Takeda, Interaction between transverse cracks and delamination during damage progress in CFRP cross-ply laminates, Composites Science and Technology 54 (4) (1995) 395-404.

[8] J. Zhang, J. Fan, K. P. Herrmann, Delaminations induced by constrained transverse cracking in symmetric composite laminates, International Journal of Solids and Structures 36 (1999) 813-846. 
[9] L. Selvakumaran, G. Lubineau, Electrical behavior of laminated composites with intralaminar degradation: a comprehensive micro-meso homogenization procedure, Composite Structures 109 (2014) 178--188.

[10] G. Lubineau, H. Nouri, F. Roger, On micro-meso relations homogenizing electrical properties of tranversely cracked laminated composites, Composite Structures 105 (2013) 66--74.

[11] L. Selvakumaran, et al., On the detectability of transverse cracks in laminated composites through measurements of electrical potential change, Composite Structures 121 (2015) 237--246.

[12] J. C. Abry, S. Bochard, A. Chateauminois, M. Salvia, G. Giraud, In-situ detection of damage in CFRP laminates by electrical resistance measurements, Composites Science and Technology 59 (1999) 925--935.

[13] A. Kaddour, et al., Electrical resistance measurement technique for detecting failure in CFRP materials at high strain rates, Composites Science and Technology 51 (1994) 377--385.

[14] A. Todoroki, M. Tanaka, Y. Shimamura, Measurement of orthotropic electric conductance of CFRP laminates and analysis of the effect on delamination monitoring with an electric resistance change method, Composites Science and Technology 62 (2002) 619--628.

[15] A. Todoroki, M. Tanaka, Y. Shimamura, Effects with a matrix crack on monitoring by electrical resistance method, Advanced Composite Materials 13 (2) (2004) 107--120.

[16] Torayca, Torayca data sheet (2005).

[17] Hextow, Hextow carbon fiber product data (February 2014).

[18] C. May (Ed.), Epoxy resins: chemistry and technology, CRC press, 1987.

[19] DOW, Dow liquid epoxy resins, Tech. rep., DOW Plastics (1999).

[20] D. Hartman, M. E. Greenwood, D. M. Miller, High strength glass fibers, Tech. rep., AGY (1996).

[21] D. Zhang, L. Ye, S. Deng, J. Zhang, Y. Tang, Y. Chen, CF/EP composite laminates with carbon black and copper chloride for improved electrical conductivity and interlaminar fracture toughness, Composites Science and Technology 72 (3) (2012) 412--420.

[22] V. Kostopoulos, et al., Damage monitoring of carbon fiber reinforced laminates using resistance measurements. improving sensitivity using carbon nanotube doped epoxy matrix system, Journal of Intelligent Material Systems and Structures 20 (2009) 1025--1034.

[23] D. Zhang, L. Ye, D. Wang, Y. Tang, S. Mustapha, Y. Chen, Assessment of transverse impact damage in GF / EP laminates of conductive nanoparticles using electrical resistivity tomography, Composites Part A 43 (9) (2012) 1587--1598.

[24] A. Markov, B. Fiedler, K. Schulte, Electrical conductivity of carbon black/fibers filled glass fiber reinforced thermoplastic composites, Composites Part A 37 (2006) 1390--1395. 